Proceedings of the Edinburgh Mathematical Society (2005) 48, 571-583 (C)

DOI:10.1017/S0013091504000495 Printed in the United Kingdom

\title{
UNIVERSAL LAURENT SERIES
}

\author{
G. COSTAKIS $^{1 *}$, V. NESTORIDIS ${ }^{1}$ AND I. PAPADOPERAKIS ${ }^{2}$ \\ ${ }^{1}$ Department of Mathematics, Athens University, \\ 15784 Panepistimiopolis, Athens, Greece (vnestor@math.uoa.gr) \\ ${ }^{2}$ Department of Mathematics, Agricultural University of Athens, \\ Iera Odos 75, Athens 118 55, Greece (gmat3pag@auadec.aua.gr)
}

(Received 4 June 2004)

\begin{abstract}
Universal Taylor series are defined on simply connected domains, but they do not exist on an annulus. Instead we introduce universal Laurent or Laurent-Faber series on finitely connected domains in $\mathbb{C}$. These are generic universalities. Furthermore, we study some properties of universal Laurent series on an annulus.
\end{abstract}

Keywords: universal series; generic property; Laurent series; Faber series

2000 Mathematics subject classification: Primary 30B30; 30B41

\section{Introduction}

If $\sum_{n=0}^{\infty} \alpha_{n} z^{n}$ has radius of convergence $R=1$, then for $\left|z_{0}\right|>1$ the sequence $S_{n}\left(z_{0}\right)=\sum_{l=0}^{n} \alpha_{l} z_{0}^{l}$ is not bounded; thus, there exists a strictly increasing sequence $\mu_{n} \in\{0,1,2 \ldots\}, n=1,2, \ldots$, so that $S_{\mu_{n}}\left(z_{0}\right) \rightarrow \infty$, as $n \rightarrow+\infty$. Therefore, there are two possibilities: either $\lim _{n} S_{n}\left(z_{0}\right)=\infty$, or there exists another strictly increasing sequence $\lambda_{n} \in\{0,1,2, \ldots\}, n=1,2, \ldots$, such that the sequence $S_{\lambda_{n}}\left(z_{0}\right)$ has a finite limit (in $\mathbb{C}$ ). In this last case, we say that the series $\sum_{n=0}^{\infty} \alpha_{n} z^{n}$ overconverges at $z_{0}$. It is also possible to replace $\left\{z_{0}\right\}$ by a compact set $K \subset\{z \in \mathbb{C}:|z| \geqslant 1\}$ ( $K^{\mathrm{c}}$ connected) and still the sequence $S_{\lambda_{n}}$ converges uniformly on $K$ towards to a function $g: K \rightarrow \mathbb{C}$. In this case we say that $g$ is obtained by overconvergence by $\sum_{n=0}^{\infty} \alpha_{n} z^{n}$. Obviously, the function $g$ is continuous on $K$ and holomorphic in $K^{0}$, since $g$ is a uniform limit of polynomials.

A power series $\sum_{n=0}^{\infty} \alpha_{n} z^{n}$ converging in $D=\{z:|z|<1\}$ is called a universal Taylor series (in $D$ ), if we can obtain by overconvergence every function $g: K \rightarrow \mathbb{C}$ that is continuous on $K$ and holomorphic in $K^{0}$ for all compact sets $K \subset \mathbb{C}$ satisfying $K \cap D=\emptyset$ and with $K^{\mathrm{c}}$ connected.

The set $U(D)$ of universal Taylor series in $D$ has been proved to be non-void and especially $G_{\delta}$ (countable intersection of open sets) and dense in $H(D)$ endowed with the topology of uniform convergence on compacta [11].

* Present address: Department of Mathematics, University of Crete, Heraklion, Knossos Ave, GR71409, Heraklion, Crete, Greece (costakis@math.uoc.gr). 
Furthermore, some properties of such universal Taylor series have been investigated in $[6-8,11]$.

In the above consideration, the open unit disc may be replaced by any simply connected domain $\Omega \subset \mathbb{C}, \Omega \neq \mathbb{C}$, and several kinds of universal Taylor series can be defined $[\mathbf{7}, \mathbf{1 2}]$. However, if $\Omega$ is an annulus, universal Taylor series do not exist $[\mathbf{2 , 7}$. Thus, it is natural to search for universal Laurent series. In fact such series exist on an annulus and this is a generic universality.

Properties of universal Taylor series on discs are easily transferred to universal Laurent series on an annulus (see $\S 3$ ). However, we wish to handle the case of any finitely connected domain in $\mathbb{C}$; in fact it is possible to have several universalities, all of them being generic. Thus in $\S 2$ we establish a unifying theorem.

Instead of partial sums we consider a sequence of maps $T_{n}, n=0,1,2, \ldots$, defined on $H(\Omega)$ and take values on some function space. We say that a function $f \in H(\Omega)$ is universal if the sequence $T_{n}(f), n=0,1, \ldots$, approximates a lot of functions outside $\Omega$ (see Definition 2.1 below). If the $T_{n}$ are continuous, then the set $U$ of universal functions is proved to be $G_{\delta}$ in $H(\Omega)$, endowed with the topology of uniform convergence on compacta. In order to combine Baire's theorem with Mergelyan's theorem and conclude that $U$ is also dense, we use an assumption of the following type: if $P$ is a rational function with prescribed poles, then $T_{n}(P)$ approaches $P$ for some subsequence of $n$.

In the case of simply connected domains, when $T_{n}$ are the partial sums of the Taylor development with respect to some centre, this condition obviously holds for all polynomials, since $T_{n}(P)=P$ for $n \geqslant \operatorname{deg} P$ and any polynomial $P$. Thus, the assumptions of the unifying theorem $(\S 2)$ are rather natural.

In order to apply this unifying theorem to the case of finitely connected domains $\Omega$, we observe that every $f \in H(\Omega)$ can be written as $f=f_{0}+f_{1}+\cdots+f_{k}$, where every $f_{j}$ is holomorphic in a simply connected domain $V_{j}$ of $\mathbb{C} \cup\{\infty\}$, with $V_{0} \subset \mathbb{C}$, while $\infty \in V_{1}, \ldots, \infty \in V_{k}$. We consider the Taylor development of $f_{0}$ and partial sums. For $f_{j}, j=1, \ldots, k$, we consider partial sums of Laurent expansions with centre $\alpha_{j} \notin V_{j} . T_{n}$ is taken to be a finite sum of those partial sums. Then the dependence on $f$ is continuous and $T_{n}(P)=P$ for all rational functions $P$ with poles in $\left\{\infty, \alpha_{1}, \ldots, \alpha_{k}\right\}$ and $n \geqslant \operatorname{deg} P$. Thus there exist universal Laurent series and they form a dense and $G_{\delta}$ subset of $H(\Omega)$.

The disadvantage is that $T_{n}(f)$ does not converge to $f$ in $\Omega$, unless it is an annulus. We can obtain this convergence if we consider Faber-Laurent expansions. This is possible when $\Omega$ is bounded by a finite number of analytic Jordan curves. It is also possible to have matrix transforms of the previous $T_{n}$, and still have generic universalities. Extensions of results of the present paper have been obtained by Vlachou in $[\mathbf{1 4}, \mathbf{1 5}]$, where approximation outside $\Omega$ and simultaneously inside $\Omega$ is obtained.

\section{A unification theorem}

In this section we consider domains $\Omega \subset \mathbb{C}$, such that $(\mathbb{C} \cup\{\infty\}) \backslash \Omega$ has a finite number of components $A_{0}, A_{1}, \ldots, A_{k}$, where $k \geqslant 0$. We assume that $\infty \in A_{0}$ and we fix points $\alpha_{j} \in$ $A_{j}, j=1, \ldots, k$. Let also $Y$ be a hemicompact space; that is, there exists an increasing sequence of compact subsets $Y_{p} \subset Y, p=1,2, \ldots$, such that every compact set $J \subset Y$ 
is contained in some $Y_{p}$. Let $H(\Omega)$ be the space of holomorphic functions in $\Omega$, with the topology of uniform convergence on compacta. In addition, we consider the space $C(Y \times$ $\left.\left(\mathbb{C} \backslash\left[\Omega \cup\left\{\alpha_{1}, \ldots, \alpha_{k}\right\}\right]\right)\right)$ of complex continuous functions on $Y \times\left(\mathbb{C} \backslash\left[\Omega \cup\left\{\alpha_{1}, \ldots, \alpha_{k}\right\}\right]\right)$ with the topology of uniform convergence on compacta. Both spaces are complete and metrizable; in fact, they are Fréchet spaces. We finally fix a sequence of maps

$$
T_{n}: H(\Omega) \rightarrow C\left(Y \times\left(\mathbb{C} \backslash\left[\Omega \cup\left\{\alpha_{1}, \ldots, \alpha_{k}\right\}\right]\right)\right), \quad n=0,1,2 \ldots
$$

Definition 2.1. Under the above notation and assumptions a holomorphic function $f \in H(\Omega)$ belongs to the class $U \equiv U\left(\Omega,\left\{T_{n}\right\}\right)$ if the following holds: for every compact set $K \subset \mathbb{C} \backslash\left\{\alpha_{1}, \ldots, \alpha_{k}\right\}$ with $K \cap \Omega=\emptyset$ and $K^{\text {c }}$ connected and every function $g: K \rightarrow \mathbb{C}$ continuous on $K$ and holomorphic in $K^{0}$, there exists a sequence $\lambda_{n} \in\{0,1,2, \ldots\}$, $n=1,2, \ldots$, such that for every compact set $J \subset Y$ we have

$$
\sup _{x \in J} \sup _{z \in K}\left|\left[T_{\lambda_{n}}(f)\right](x, z)-g(z)\right| \rightarrow 0 \quad \text { as } n \rightarrow+\infty .
$$

In the previous definition it is equivalent to require $\lambda_{n}<\lambda_{n+1}$.

In order to obtain a denumerable description of the class $U$ we consider $q_{j}, j=1,2, \ldots$, an enumeration of all polynomials with coefficients in $\boldsymbol{Q}+\mathrm{i} \boldsymbol{Q}$, and on the other hand we use Lemma 2.2 below.

Lemma 2.2. Let $\Omega$ be a domain in $\mathbb{C}, \Omega \neq \mathbb{C}$. We assume that $(\boldsymbol{C} \cup\{\infty\}) \backslash \Omega$ has components $A_{0}, \ldots, A_{k}, k \geqslant 0$, and we fix $\infty \in A_{0}, \alpha_{1} \in A_{1}, \ldots, \alpha_{k} \in A_{k}$. Then there exists a sequence of compact sets $K_{m} \subset \mathbb{C} \backslash\left\{\alpha_{1}, \ldots, \alpha_{k}\right\}, m=1,2, \ldots$, with $K_{m} \cap \Omega=\emptyset$ and $K_{m}^{\mathrm{c}}$ connected such that the following holds.

Every compact set $K \subset \mathbb{C} \backslash\left\{\alpha_{1}, \ldots, \alpha_{k}\right\}$ with $K \cap \Omega=\emptyset$ and $K^{\mathrm{c}}$ connected is contained in $K_{m}$, for some $m=1,2, \ldots$.

Proof. We fix a point $\zeta_{0}$ in $\Omega$. Let $K \subset \mathbb{C} \backslash\left\{\alpha_{1}, \ldots, \alpha_{k}\right\}$ be a compact set satisfying $K \cap \Omega=\emptyset$ and with $K^{\mathrm{c}}$ connected. Since $\zeta_{0}, \infty, \alpha_{1}, \ldots, \alpha_{k}$ belong to $(\mathbb{C} \cup\{\infty\}) \backslash K$, which is connected, we can find polygonal lines $\gamma_{0}, \gamma_{1}, \ldots, \gamma_{k}$ in $(C \cup\{\infty\}) \backslash K$ starting from $\zeta_{0}$ and ending at $\infty, \alpha_{1}, \ldots, \alpha_{k}$, respectively (the last edge of $\gamma_{0}$ is a closed half-line of the form $\{x \in \mathbb{R}: x \geqslant n\} \cup\{\infty\}$, for some natural number $n$ ). We assume that all other vertices of $\gamma_{0}, \gamma_{1}, \ldots, \gamma_{k}$ are in $\boldsymbol{Q}+\mathrm{i} \boldsymbol{Q}$. The set $\Gamma=\gamma_{0} \cup \gamma_{1} \cup \cdots \cup \gamma_{k}$ is compact and disjoint from $K$. If 'dist' denotes the distance in $\mathbb{C} \cup\{\infty\}$ induced by Euclidean distance in $\mathbb{R}^{3}$ via stereographical projection, then $\operatorname{dist}(\Gamma, K) \geqslant(1 / s)>0$ for some $s \in\{1,2, \ldots\}$. Thus $K \subset L(\Gamma, s)$, where

$$
L(\Gamma, s)=\left\{z \in \mathbb{C} \cup\{\infty\}: z \in \Omega^{\mathrm{c}} \text { and } \operatorname{dist}(z, \Gamma) \geqslant(1 / s)\right\} .
$$

The set $L(\Gamma, s)$ is compact in $\mathbb{C} \backslash\left\{\alpha_{1}, \ldots, \alpha_{k}\right\}$ and is disjoint from $\Omega$, its complement is

$$
\Omega \cup\{z \in C \cup\{\infty\}: \operatorname{dist}(z, \Gamma)<(1 / s)\},
$$

which is connected as the union of two connected sets containing the same point $\zeta_{0}$.

Since the collection of all $L(\Gamma, s)$ is denumerable, an enumeration of them gives the desired sequence $K_{m}, m=1,2, \ldots$. 
We fix a sequence $K_{m}, m=1,2, \ldots$, as in Lemma 2.2.

Also let $L_{p}, p=1,2, \ldots$, be an exhausting family of compact sets in $\Omega$. By our assumption in $\Omega$, we can assume that $(\mathbb{C} \cup\{\infty\}) \backslash L_{p}$ has $k+1$ components, one containing $\infty$ and the others $\alpha_{1}, \ldots, \alpha_{k}$, respectively [13, Theorem 13.3].

For $J \subset Y$ compact, $K \subset \Omega^{\mathrm{c}}$ compact with $K^{\mathrm{c}}$ connected, $g$ a complex function on $K, n \in\{0,1,2, \ldots\}$ and $s \in\{1,2, \ldots\}$ we consider the set

$$
E(\Omega, J, K, g, n, s)=\left\{f \in H(\Omega): \sup _{x \in J} \sup _{z \in K}\left|\left[T_{n}(f)\right](x, z)-g(z)\right|<(1 / s)\right\} .
$$

Proposition 2.3. Under the above assumptions and using the same notation we have

$$
U=\bigcap \bigcap_{p} \bigcap_{j} \bigcup_{s}^{\infty} E\left(\Omega, Y_{p}, K_{m}, q_{j}, n, s\right) .
$$

The proof of Proposition 2.3 is based on Mergelyan's theorem [13] and is similar to the proof of Lemma 3.5 of [7].

In order to prove that $U$ is $G_{\delta}$ and dense in the complete space $H(\Omega)$, Baire's theorem can be applied. It suffices to prove that under some conditions, the set $E\left(\Omega, Y_{p}, K_{m}, q_{j}, n, s\right)$ is open and that $\bigcup_{n=0}^{\infty} E\left(\Omega, Y_{\rho}, K_{m}, q_{j}, n, s\right)$ is dense in $H(\Omega)$.

Proposition 2.4. Under the above assumptions and using the same notation, we also assume that the maps $T_{n}: H(\Omega) \rightarrow C\left(Y \times\left(\mathbb{C} \backslash\left[\Omega \cup\left\{\alpha_{1}, \ldots \alpha_{k}\right\}\right]\right)\right)$ are continuous. Then $E\left(\Omega, Y_{p}, K_{m}, q_{j}, n, s\right)$ is open in $H(\Omega)$ and the class $U$ is $G_{\delta}$ in $H(\Omega)$.

For the proof it suffices to observe that the set

$$
A=\left\{\omega \in C\left(Y \times\left(\mathbb{C} \backslash\left[\Omega \cup\left\{a_{1}, \ldots, a_{k}\right\}\right]\right)\right): \sup _{x \in Y_{p}} \sup _{z \in K_{m}}\left|\omega(x, z)-q_{j}(z)\right|<(1 / s)\right\}
$$

is open and $T_{n}^{-1}(A)=E\left(\Omega, Y_{p}, K_{m}, q_{j}, n, s\right)$. In order to establish that

$$
\bigcup_{n=0}^{\infty} E\left(\Omega, Y_{p}, K_{m}, q_{j}, n, s\right)
$$

is dense in $H(\Omega)$, we make the assumption that the applications $T_{n}$ 'respect in the limit' each rational function whose poles are included in $\left\{\infty, \alpha_{1}, \ldots, \alpha_{k}\right\}$.

Theorem 2.5. Let $\Omega \subset \mathbb{C}$ be a domain of finite connectivity with $\infty, \alpha_{1}, \ldots, \alpha_{k}$ fixed points in each component of $(\mathbb{C} \cup\{\infty\}) \backslash \Omega$. Let $Y$ be a hemicompact topological space. We endow the spaces $H(\Omega)$ and $C\left(Y \times\left(\mathbb{C} \backslash\left[\Omega \cup\left\{\alpha_{1}, \ldots, \alpha_{k}\right\}\right]\right)\right)$ with the topology of uniform convergence on compacta. We consider a sequence of maps $T_{n}: H(\Omega) \rightarrow$ $C\left(Y \times\left(\mathbb{C} \backslash\left[\Omega \cup\left\{\alpha_{1}, \ldots \alpha_{k}\right\}\right]\right)\right)$ and the corresponding class $U$ defined in Definition 2.1.

We assume that the $T_{n}$ are continuous and that there exists a function $h: \mathbb{C} \backslash[\Omega \cup$ $\left.\left\{\alpha_{1}, \ldots, \alpha_{k}\right\}\right] \rightarrow \mathbb{C}$ continuous on its domain of definition and holomorphic on the interior, satisfying $h(z) \neq 0$ for all $z$ in $\mathbb{C} \backslash\left[\Omega \cup\left\{\alpha_{1}, \ldots, \alpha_{2}\right\}\right]$, such that the following holds. 
For every rational function $P$, whose poles are included in $\left\{\infty, \alpha_{1}, \ldots, \alpha_{k}\right\}$, and every compact set $K \subset \mathbb{C}$ with $K \cap\left[\Omega \cup\left\{\alpha_{1}, \ldots, \alpha_{k}\right\}\right]=\emptyset$ and $K^{\mathrm{c}}$ connected there exists a sequence $n_{\tau} \in\{0,1,2, \ldots\}, \tau=1,2, \ldots$, such that, for every compact set $J \subset Y$ we have

$$
\sup _{x \in J} \sup _{z \in K}\left|\left[T_{n_{\tau}}(P)\right](x, z)-h(z) P(z)\right| \rightarrow 0 \quad \text { as } \tau \rightarrow \infty .
$$

Then the class $U$ is $G_{\delta}$ and dense in $H(\Omega)$.

Proof. Combining Baire's theorem with Propositions 2.3 and 2.4 it suffices to prove that $\bigcup_{n=0}^{\infty} E(\Omega, J, K, g, n, s)$ is dense in $H(\Omega)$ for every compact set $J \subset Y, K \subset \mathbb{C}$ compact with $K^{\mathrm{c}}$ connected and $K \cap\left[\Omega \cup\left\{\alpha_{1}, \ldots, \alpha_{k}\right\}\right]=\emptyset, g: K \rightarrow \mathbb{C}$ continuous on $K$ and holomorphic in $K^{0}$ and $s \in\{1,2, \ldots\}$.

Let $f \in H(\Omega), L \subset \Omega$ compact and $\varepsilon>0$. We consider an open neighbourhood $V(f)$ of $f$ in $H(\Omega)$ of the form $V(f)=\left\{P \in H(\Omega): \sup _{w \in L}|P(w)-f(w)|<\varepsilon\right\}$. Without loss of generality we may assume that $(\mathbb{C} \cup\{\infty\}) \backslash L$ has $k+1$ components for some $k \geqslant 0$, each one containing $\infty, \alpha_{1}, \ldots, \alpha_{k}$, respectively. We have to prove that $V(f)$ meets $\bigcup_{n=0}^{\infty} E(\Omega, J, K, g, n, s)$ : that is, find $P \in H(\Omega)$ and $n \geqslant 0$ such that $P \in V(f)$ and $P \in E(\Omega, J, K, g, n, s)$.

We set $M=\sup _{z \in K}|h(z)| \in(0,+\infty)$ and we observe that the function $g / h$ is continuous on $K$ and holomorphic in $K^{0}$. Since $L$ and $K$ are disjoint compact sets, our assumption on the number of components of their complements in $\mathbb{C} \cup\{\infty\}$ implies that $L \cup K$ is a compact set in $\mathbb{C}$ and $(\mathbb{C} \cup\{\infty\}) \backslash(L \cup K)$ has $k+1$ components, each one containing $\infty, \alpha_{1}, \ldots, \alpha_{k}$, respectively. We consider the function $F: L \cup K \rightarrow \mathbb{C}$ defined by $F(w)=f(w)$ for $w \in L$ and $F(z)=(g(z) / h(z))$ for $z \in K$. By an extension of Mergelyan's theorem [13, Exercise 1, p. 394], there exists a rational function $P$ whose poles are included in $\left\{\infty, \alpha_{1}, \ldots, \alpha_{k}\right\}$ such that

$$
\sup _{w \in L}|P(w)-f(w)|<\varepsilon
$$

and

$$
\sup _{z \in K}\left|P(z)-\frac{g(z)}{h(z)}\right|<\frac{1}{2 s M}
$$

which implies

$$
\sup _{z \in K}|h(z) P(z)-g(z)|<\frac{1}{2 s} .
$$

By assumption there exists $n_{\tau} \in\{0,1,2, \ldots\}$ such that

$$
\sup _{x \in J} \sup _{z \in K}\left|\left[T_{n_{\tau}}(P)\right](x, z)-h(z) P(z)\right|<\frac{1}{2 s} \text {. }
$$

We set $n=n_{\tau}$. 
First, $P$ belongs to $H(\Omega)$, since it is rational with poles outside of $\Omega$, and $P \in V(f)$ by $(*)$. Further we have

$$
\begin{aligned}
\sup _{x \in J} \sup _{z \in K} \mid\left[T_{n}(P)\right](x, z) & -g(z) \mid \\
& \leqslant \sup _{x \in J} \sup _{z \in K}\left|\left[T_{n_{\tau}}(P)\right](x, z)-h(z) P(z)\right|+\sup _{z \in K}|h(z) P(z)-g(z)| \\
& <\frac{1}{2 s}+\frac{1}{2 s}=\frac{1}{s},
\end{aligned}
$$

where we have used relations $(* * *)$ and $(* *)$. This completes the proof.

\section{The case of an annulus}

We consider the special case where $\Omega$ is an annulus $\Delta=\Delta\left(0, R_{1}, R_{2}\right)=\{z \in \mathbb{C}$ : $\left.R_{1}<|z|<R_{2}\right\}$ with $0<R_{1}<R_{2}<+\infty$. We set $\alpha_{1}=0$ and $Y$ to be a singleton. If $f(z)=\sum_{n=-\infty}^{+\infty} \alpha_{n}(f) z^{n}$ belongs to $H(\Delta)$, we set $T_{n}(f)(z)=\sum_{k=-n}^{n} \alpha_{k}(f) z^{k}$. We denote by $U(\Delta)$ the class $U\left(\Delta,\left\{T_{n}\right\}\right)$ defined in Definition 2.1. It is also easy to see that the assumptions of Theorem 2.5 are satisfied in this case. Therefore, the class $U(\Delta)$ is dense and $G_{\delta}$ in $H(\Delta)$.

We also need the notion of universal Taylor series in a disc introduced in [11].

Definition 3.1. Let $D_{R}=\{z \in \mathbb{C}:|z|<R\}, 0<R<+\infty$, be a disc. A holomorphic function $f(z)=\sum_{n=0}^{+\infty} \delta_{n} z^{n}$ in $H\left(D_{R}\right)$ belongs to the class $U\left(D_{R}\right)$ if the following holds.

For every compact set $K \subset \mathbb{C}, K \cap D_{R}=\emptyset$ with $K^{\mathrm{c}}$ connected and every function $g: K \rightarrow \mathbb{C}$ continuous on $K$ and holomorphic in $K^{0}$, there exists a sequence $\lambda_{n} \in$ $\{0,1,2, \ldots\}, n=1,2, \ldots$, such that

$$
\sup _{z \in K}\left|\sum_{k=0}^{\lambda_{n}} \delta_{k} z^{k}-g(z)\right| \rightarrow 0 \quad \text { as } n \rightarrow+\infty .
$$

In the above definition it is equivalent to require $\lambda_{n}<\lambda_{n+1}$.

The set $U\left(D_{R}\right)$ has been proved to be $G_{\delta}$ and dense in $H\left(D_{R}\right)$ [11]. Properties of the elements of $U\left(D_{R}\right)$ have been established in $[\mathbf{1}, \mathbf{6}-\mathbf{8}]$. In the rest of this section we investigate properties of the elements of the class $U(\Delta)$.

Remark 3.2. Let

$$
\sum_{k=-\infty}^{+\infty} c_{k} z^{k} \in U(\Delta), \quad \Delta=\Delta\left(0, R_{1}, R_{2}\right) .
$$

Then

$$
\sum_{k=0}^{\infty} c_{k} z^{k} \in U\left(D_{R_{2}}\right) \quad \text { and } \quad \sum_{k=1}^{\infty} c_{-k} w^{k} \in U\left(D_{1 / R_{1}}\right) .
$$

This is obvious if we recall that $\sum_{k=1}^{\infty} c_{k} z^{k}$ converges uniformly on compacta of $|z|<R_{2}$ and $\sum_{k=-\infty}^{-1} c_{k} z^{k}$ converges uniformly on compacta of $|z|>R_{1}$. 
Remark 3.3. For every

$$
\sum_{l=-\infty}^{\infty} c_{l} z^{l} \in U(\Delta), \quad \Delta=\Delta\left(0, R_{1}, R_{2}\right)
$$

and every $z_{0}$ with $\left|z_{0}\right|=R_{1}$ or $\left|z_{0}\right|=R_{2}$, the series $\sum_{l=-\infty}^{+\infty} c_{l} z_{0}^{l}$ is not $(C, k)$ summable for any $k=1,2, \ldots$.

If $\left|z_{0}\right|=R_{2}$ we observe that $\sum_{l=1}^{\infty} c_{-l} z_{0}^{-l}$ converges and that $\sum_{l=0}^{\infty} c_{k} z^{l} \in U\left(D_{R_{2}}\right)$, and then, using the corresponding property of a universal Taylor series on a disc $[\mathbf{7}, \mathbf{8}]$, the result follows.

If $u \in U\left(D_{R}\right)$ and $\left|z_{0}\right|=R$, it is also true that

$$
\lim _{\substack{\zeta \rightarrow z_{0} \\ z \in D_{R}}} u(z)
$$

does not exist in $\mathbb{C}[\mathbf{7}]$.

Thus we obtain the following.

Remark 3.4. If $f \in U(\Delta), \Delta=\Delta\left(0, R_{1}, R_{2}\right)$, and $z_{0} \in \partial \Delta$, then

$$
\lim _{\substack{\zeta \rightarrow z_{0} \\ z \in \mathcal{D}}} f(z)
$$

does not exist in $\mathbb{C}$. In particular, $f$ cannot be continuously extended in $\bar{\Delta}$ and $f$ cannot be holomorphically extended in any domain strictly containing $\Delta$.

Remark 3.5. Let

$$
\sum_{k=-\infty}^{+\infty} c_{k} z^{k} \in U(\Delta), \quad \Delta=\Delta\left(0, R_{1}, R_{2}\right),
$$

and let $g_{1}$ and $g_{2}$ be two complex-valued measurable $2 \pi$-periodic functions. Then there exists a sequence $0 \leqslant \lambda_{n}<\lambda_{n+1}$ such that

(i) $\lim _{n} \sum_{k=-\lambda_{n}}^{\lambda_{n}} c_{k} R_{2}^{k} \mathrm{e}^{\mathrm{i} k \vartheta}=g_{2}(\vartheta)$ and

(ii) $\lim _{n} \sum_{k=-\lambda_{n}}^{\lambda_{n}} c_{k} R_{1}^{k} \mathrm{e}^{\mathrm{i} k \vartheta}=g_{1}(\vartheta)$

for almost all $\vartheta$.

It suffices to use Lusin's theorem to approximate (in the almost everywhere sense) $g_{1}$ and $g_{2}$ by continuous functions $\varphi_{n}^{1}, \varphi_{n}^{2}$ and then apply the definition of $U(\Delta)$ to the compact sets

$$
K_{n}=\left\{z \in \mathbb{C} ;|z|=R_{2}, \frac{1}{n} \leqslant \arg z \leqslant 2 \pi\right\} \cup\left\{z \in \mathbb{C}:|z|=R_{1}, \frac{1}{n} \leqslant \arg z \leqslant 2 \pi\right\}
$$

(see also $[\mathbf{1 1}])$. 
The above is an extension of Menchoff's result on universal trigonometric series $[\mathbf{3}, \mathbf{7}, \mathbf{9}]$.

Remark 3.6. Let

$$
\sum_{k=-\infty}^{+\infty} c_{k} z^{k} \in U(\Delta), \quad \Delta=\Delta\left(0, R_{1}, R_{2}\right) .
$$

Then we have the following.

(i) $\lim \sup _{k \rightarrow+\infty} \sqrt[k]{\left|c_{k}\right|}=1 / R_{2}$.

(ii) $\lim \sup _{k \rightarrow+\infty} \sqrt[k]{\left|c_{-k}\right|}=R_{1}$.

(iii) For every decreasing sequence $b_{n} \downarrow 0$ such that $\sum_{n=1}^{\infty}\left(b_{n} / n\right)<+\infty$ we have

$$
\limsup _{k \rightarrow+\infty} \frac{\left|c_{n}\right| R_{2}^{n}}{\mathrm{e}^{n b_{n}}}=+\infty \text {. }
$$

In particular, $c_{n} R_{2}^{n}$ does not have polynomial growth, as $n \rightarrow+\infty$.

(iv) For every decreasing sequence $b_{n} \downarrow 0$, such that $\sum_{n=1}^{\infty}\left(b_{n} / n\right)<+\infty$ we have

$$
\lim _{n \rightarrow+\infty} \frac{\left|c_{-n}\right|}{R_{1}^{n} \mathrm{e}^{n b_{n}}}=+\infty
$$

In particular, $c_{-n} / R_{1}^{n}$ does not have polynomial growth, as $n \rightarrow+\infty$.

The proof follows from $[\mathbf{8}]$ and from the fact that

$$
\sum_{k=0}^{\infty} c_{k} z^{k} \in U\left(\mathcal{D}_{R_{2}}\right) \quad \text { and } \quad \sum_{k=1}^{\infty} c_{-k} w^{k} \in U\left(D_{1 / R_{1}}\right) .
$$

Finally, we have a great Picard type property.

The proof follows the lines of $[\mathbf{1}]$.

Proposition 3.7. Let $f \in H(\Delta)$, where $\Delta=\Delta(0, R, 1), 0<R<1$, and let $n$ be a non-negative integer. If the holomorphic functions $f$ and $f^{(n)}-1$ have at most finitely many zeros in $\Delta$, then $f \notin U(\Delta)$.

Proof. Let $f$ satisfy the hypotheses of this proposition. Then, there is $0<p_{0}<1$ with $p_{0}>R$ such that $f$ and $f^{(n)}-1$ have no zeros in the annulus $\Delta\left(0, p_{0}, 1\right)$. Let $p=\frac{1}{2}\left(1+p_{0}\right), \delta=\frac{1}{2}\left(1-p_{0}\right)>0$ and fix $z_{0}=p \mathrm{e}^{\mathrm{i} \vartheta}$. Define

$$
F(z)=\delta^{-n} f\left(z_{0}+\delta z\right) \text { for } z \in D=\{z \in \mathbb{C}:|z|<1\} .
$$

Then $F$ and $F^{(n)}-1$ have no zeros in $D$. Hence, by Miranda's theorem [10] we conclude that

$$
\log |F(z)| \leqslant \frac{1}{1-|z|}\left[64 \log ^{+}|F(0)|+\frac{M_{n}}{(1-|z|)^{4}}\right] \text { for every } z \in D,
$$


where $M_{n}$ depends only on $n$. Let $p_{0}<r<1$ be such that $1-r<\delta$ and take $z=r_{1} \mathrm{e}^{\mathrm{i} \vartheta}$ in the above inequality where $r_{1} \in(0,1)$ is such that $\delta\left(1-r_{1}\right) 1-r$. We have $F\left(r_{1} \mathrm{e}^{\mathrm{i} \vartheta}\right)=$ $\delta^{-n} f\left(r \mathrm{e}^{\mathrm{i} \theta}\right)$ and there is a constant $c_{1}$ depending only on $\delta$ and $n$ such that

$$
\log \left|f\left(r \mathrm{e}^{\mathrm{i} \theta}\right)\right| \leqslant \frac{c_{1}}{1-r}\left[\log ^{+}\left|f\left(p \mathrm{e}^{\mathrm{i} \theta}\right)\right|+\frac{1}{(1-r)^{4}}\right]
$$

whenever $0<1-r<\delta$ and $p_{0}<r<1$.

Thus $\log \left|f\left(r \mathrm{e}^{\mathrm{i} \theta}\right)\right| \leqslant c /(1-r)^{5}$ for all $r \in(0,1)$ such that $0<1-r<\delta$ and $p_{0}<r<1$. Hence the proof will be completed after the following lemma is established, which follows by a combination of Remark 3.6 of the present paper and Lemma 2.2 of [1].

Lemma 3.8. Let $c, k$ be positive real numbers and $0<R<1$. Then for every $f \in H(\Delta(0, R, 1))$ such that

$$
|f(z)|=O\left(\mathrm{e}^{c /(1-|z|)^{k}}\right) \quad \text { as }|z| \rightarrow 1
$$

we have $f \notin U(\Delta(0, R, 1))$.

And the proof is complete.

Remark 3.9. The previous proof implies easily that if $u \in U\left(\Delta\left(0, R_{1}, R_{2}\right)\right)$, then for every $\alpha \in \mathbb{C}$ with at most one exception the following holds: the roots of the equation $u(z)=\alpha, R_{1}<|z|<R_{2}$, have at least two cluster points $z_{1}, z_{2}$ with $\left|z_{1}\right|=R_{1}$ and $\left|z_{2}\right|=R_{2}$.

\section{General Laurent expansions}

Let $\Omega$ be a domain in $\mathbb{C}$, such that $(\mathbb{C} \cup\{\infty\}) \backslash \Omega$ has a finite number of components $A_{0}, A_{1}, \ldots, A_{k}(k \geqslant 0)$ with $\infty \in A_{0}, \alpha_{1} \in A_{1}, \ldots, \alpha_{k} \in A_{k}$ being fixed.

We consider the space $H\left(A_{0}^{\mathrm{c}}\right)$ with the topology of uniform convergence on compacta. For $j \in\{1, \ldots, k\}$ let $H_{0}\left(A_{j}^{\mathrm{c}}\right)$ be the subspace of $H\left(A_{j}^{\mathrm{c}}\right)$ consisting of all $f \in H\left(A_{j}^{\mathrm{c}}\right)$ satisfying $f(\infty)=0$. We endow $H_{0}\left(A_{j}^{\mathrm{c}}\right)$ with the topology of uniform convergence on compacta. All spaces $H\left(A_{0}^{\mathrm{c}}\right), H_{0}\left(A_{1}^{\mathrm{c}}\right), \ldots, H_{0}\left(A_{k}^{\mathrm{c}}\right)$ are Fréchet spaces.

Since $\Omega=A_{0}^{\mathrm{c}} \cap A_{1}^{\mathrm{c}} \cap \cdots \cap A_{k}^{\mathrm{c}}$ we can consider the map

$$
T: H\left(A_{0}^{\mathrm{c}}\right) \times H_{0}\left(A_{1}^{\mathrm{c}}\right) \times \cdots \times H_{0}\left(A_{k}^{\mathrm{c}}\right) \rightarrow H(\Omega)
$$

defined by $T\left(f_{0}, f_{1}, \ldots, f_{k}\right)=f_{0}+f_{1}+\cdots+f_{k}$.

Obviously, $T$ is linear and continuous (where $H(\Omega)$ is endowed with the Fréchet topology of uniform convergence on compacta). Liouville's theorem implies easily that $T$ is one to one. We will show that $T$ is also onto (hence an isomorphism).

Let $f \in H(\Omega)$ and $z \in A_{0}^{\mathrm{c}}$. We consider any cycle $\gamma_{0, z}$ in $\Omega$, such that $\operatorname{ind}\left(\gamma_{0, z}, z\right)=1$, $\operatorname{ind}\left(\gamma_{0, z}, \alpha_{j}\right)=1$ for all $j=1, \ldots, k$ and obviously ind $\left(\gamma_{0, z}, \infty\right)=0$. Then the integral

$$
f_{0}(z)=\frac{1}{2 \pi \mathrm{i}} \int_{\gamma_{0, z}} \frac{f(\zeta)}{\zeta-z} \mathrm{~d} \zeta
$$


is independent of the choice of the cycle $\gamma_{0, z}$ and defines a holomorphic function $f_{0} \in$ $H\left(A_{0}^{\mathrm{c}}\right)$; thus $f_{0}$ is given by a Cauchy transform on varying cycles.

Let $j_{0} \in\{1, \ldots, k\}, f \in H(\Omega)$ and $z \in A_{j_{0}}^{\text {c }}$; we consider any cycle $\gamma_{j_{0}, z}$ in $\Omega$, such that

$$
\operatorname{ind}\left(\gamma_{j_{0}, z}, z\right)=0, \quad \operatorname{ind}\left(\gamma_{j_{0}, z}, a_{j_{0}}\right)=-1, \quad \operatorname{ind}\left(\gamma_{j_{0}, z}, \alpha_{j}\right)=0
$$

for all $j \in\{1, \ldots, k\} \backslash\left\{j_{0}\right\}$ and obviously $\operatorname{ind}\left(\gamma_{j_{0}, z}, \infty\right)=0$. Then the integral

$$
f_{j_{0}}(z)=\frac{1}{2 \pi \mathrm{i}} \int_{\gamma_{j_{0}, z}} \frac{f(\zeta)}{\zeta-z} \mathrm{~d} \zeta
$$

is independent of the choice of the cycle $\gamma_{j_{0}, z}$ and defines a holomorphic function $f_{j_{0}} \in H_{0}\left(A_{j_{0}}^{\mathrm{c}}\right)$; thus $f_{j_{0}}$ is defined by a Cauchy transform on varying cycles.

Cauchy's theorem implies that for $z \in \Omega$ we have $f(z)=f_{0}(z)+f_{1}(z)+\cdots+f_{k}(z)$. Thus we have proved the following proposition.

Proposition 4.1. The operator $T: H\left(A_{0}^{\mathrm{c}}\right) \times H_{0}\left(A_{1}^{\mathrm{c}}\right) \times \cdots \times H_{0}\left(A_{k}^{\mathrm{c}}\right) \rightarrow H(\Omega)$ is an isomorphism. If $f \in H(\Omega), f_{0} \in H\left(A_{0}^{\mathrm{c}}\right), f_{1} \in H_{0}\left(A_{1}^{\mathrm{c}}\right), \ldots, f_{k} \in H_{0}\left(A_{k}^{\mathrm{c}}\right)$ are related by $f=f_{0}+f_{1}+\cdots+f_{k}$, then $f_{0}, f_{1}, \ldots, f_{k}$ are called the components of $f$ and depend continuously on $f$.

In view of Theorem 2.5 the map $T_{n}$ can be constructed as a sum of quantities, each one varying continuously on $f_{0}, f_{1}, \ldots, f_{k}$, respectively.

We restrict our attention to the following example.

Let $\phi_{0}:\{0,1, \ldots\} \rightarrow\{0,1, \ldots\}$ be such that $\lim _{n \rightarrow+\infty} \phi_{0}(n)=+\infty$ and let $\zeta \in A_{0}^{\mathrm{c}}$. Then for $Y=\{\zeta\}$ or $Y=A_{0}^{\mathrm{c}}$ we set

$$
T_{n}^{0}\left(f_{0}\right)=\sum_{l=0}^{\phi_{0}(n)} \frac{f_{0}^{(l)}(\zeta)}{l !}(z-\zeta)^{l} .
$$

Let $j_{0} \in\{1, \ldots, k\}$ and $\phi_{j_{0}}:\{0,1, \ldots\} \rightarrow\{0,1, \ldots\}$ be such that $\lim _{n \rightarrow+\infty} \phi_{j_{0}}(n)=+\infty$. The function $f_{j_{0}}$ has a Laurent expansion for $\left|z-\alpha_{j_{0}}\right|$ large of the form

$$
f_{j_{0}}(z)=\sum_{-\infty}^{-1} c_{l}\left(f_{j_{0}}\right)\left(z-\alpha_{j_{0}}\right)^{l},
$$

where $c_{l}\left(f_{j_{0}}\right)$ depends continuously on $f_{j_{0}}$ by the well-known formula for Laurent coefficients. We set

$$
T_{n}^{j_{0}}\left(f_{j_{0}}\right)(z)=\sum_{l=-1}^{-\phi_{j_{0}}(n)} c_{l}\left(f_{j_{0}}\right)\left(z-\alpha_{j_{0}}\right)^{l} .
$$

Finally, setting $T_{n}(f)=T_{n}^{0}\left(f_{0}\right)+T_{n}^{1}\left(f_{1}\right)+\cdots+T_{n}^{k}\left(f_{k}\right)$ one can easily check that the assumptions of Theorem 2.5 are satisfied with $h \equiv 1$. Thus we have the following theorem.

Theorem 4.2. Under the above assumptions and notation, the set of Laurent universal series $U\left(\Omega,\left\{T_{n}\right\}\right)$ is a $G_{\delta}$ and dense subset of $H(\Omega)$. 
Remark 4.3. The operators $T_{n}^{0}\left(f_{0}\right), T_{n}^{j_{0}}\left(f_{j_{0}}\right)$ may be replaced by Cesàro means depending continuously on a parameter $\alpha \in(-1,+\infty)$ (see [7]); then the parameter space $Y$ may be $(-1,+\infty) \times\{\zeta\}$ or $(-1,+\infty) \times\left(A_{0}^{\mathrm{c}}\right)$.

In all previous examples the function $h$ in the assumptions of Theorem 2.5 is identically equal to 1 . More general functions $h$ arise if we consider holomorphically varying matrix transforms in accordance with [7]; the general case of $h$ has been used in the proof of Theorem 4.3 in [7].

\section{The case of analytic boundaries}

If $\Omega=\Delta=\left\{z: R_{1}<|z|<R_{2}\right\}$ with $0<R_{1}<R_{2}<+\infty$ and $\alpha_{1}=0$, then the constructed sequence $T_{n}, n=0,1,2$, has the property that for every $f \in H(\Omega)$ the sequence $T_{n}(f)$ is also defined in $\Omega$ and converges to $f$ on $\Omega$. However, in the general setting of the previous section, $T_{n}(f)$ is also defined on $\Omega$, but it does not converge anywhere on $\Omega$. Instead, under some assumptions on $\Omega$, we will modify $T_{n}$ by means of Faber-type expansions to obtain $T_{n}(f) \rightarrow f$ on $\Omega$.

In the rest of this section we assume that $\Omega$ is a domain in $\mathbb{C}$ bounded by a finite number of analytic Jordan curves.

Let $A_{0}, A_{1}, \ldots, A_{k}$, where $k \geqslant 0$, be the components of $(\mathbb{C} \cup\{\infty\}) \backslash \Omega$ and $\infty \in A_{0}$, $\alpha_{1} \in A_{1}, \ldots, \alpha_{k} \in A_{k}$ be fixed.

If $f \in H(\Omega)$, then $f=f_{0}+f_{1}+\cdots+f_{k}$ with $f_{0} \in H\left(A_{0}^{\mathrm{c}}\right), f_{1} \in H_{0}\left(A_{1}^{\mathrm{c}}\right), \ldots$, $f_{k} \in H_{0}\left(A_{k}^{\mathrm{c}}\right)$ according to Proposition 4.1.

The simply connected domain $A_{0}^{\mathrm{c}}$ is bounded by an analytic Jordan curve; thus, if the $F_{l}^{0}, l=0,1,2, \ldots$, denote its Faber polynomials (see $[\mathbf{4}, \mathbf{5}]$ ), then $f_{0}$ has a unique Faber expansion $f_{0}(z)=\sum_{l=0}^{\infty} d_{l}\left(f_{0}\right) F_{l}^{0}(z)$ valid on the whole $A_{0}^{\mathrm{c}}$.

Let $\phi_{0}:\{0,1,2, \ldots\} \rightarrow\{0,1,2, \ldots\}$ be such that $\lim _{n} \phi_{0}(n)=+\infty$. We set

$$
T_{n}^{0}\left(f_{0}\right)=\sum_{l=0}^{\phi_{0}(n)} d_{l}\left(f_{0}\right) F_{l}^{0}(z)
$$

and $Y$ is taken to be a singleton.

Let $j_{0} \in\{1, \ldots, k\}$. Let $D_{j_{0}}$ be the image of $A_{j_{0}}^{\mathrm{c}} \cup\{\infty\}$ under the map $w=1 /\left(z-\alpha_{j_{0}}\right)$ and let the $F_{l}^{j_{0}}, l=0,1,2, \ldots$, denote the Faber polynomials of $D_{j_{0}}$; then one can easily deduce the unique expansion

$$
f_{j_{0}}(z)=\sum_{l=0}^{\infty} d_{l}^{j_{0}}\left(f_{j_{0}}\right) F_{l}^{j_{0}}\left(\frac{1}{z-\alpha_{j_{0}}}\right)
$$

valid in $A_{j_{0}}^{\mathrm{c}} \cup\{\infty\}$.

Let $\phi_{j_{0}}:\{0,1,2, \ldots\} \rightarrow\{0,1,2, \ldots\}$ be such that $\lim _{n} \phi_{j_{0}}(n)=+\infty$. Then we set

$$
T_{n}^{j_{0}}\left(f_{j_{0}}\right)(z)=\sum_{l=0}^{\phi_{j_{0}}(n)} d_{l}^{j_{0}}\left(f_{j_{0}}\right) F_{l}^{j_{0}}\left(\frac{1}{z-\alpha_{j_{0}}}\right) .
$$


Finally, we set $T_{n}(f)=T_{n}^{0}\left(f_{0}\right)+T_{n}^{1}\left(f_{1}\right)+\cdots+T_{n}^{k}\left(f_{k}\right)$ and using the results of [4] we can easily check that the assumptions of Theorem 2.5 are satisfied with $h \equiv 1$. It is also true that $T_{n}(f)(z) \rightarrow f(z)$ uniformly on compact subsets of $\Omega$, for all $f \in H(\Omega)$.

The universal functions $U\left(\Omega,\left\{T_{n}\right\}\right)$ are called universal Faber-Laurent series; their set is a $G_{\delta}$ and dense subset of $H(\Omega)$, provided that $\Omega$ is a domain bounded by a finite number of analytic Jordan curves.

It is also possible to use matrix transforms or to use Faber, Taylor and Laurent expansions simultaneously, and still have generic universalities.

Using properties of universal Faber series in simply connected domains bounded by an analytic curve [4], one can easily deduce the following proposition.

Proposition 5.1. Let $z_{0} \in \partial \Omega$ be any boundary point of $\Omega$. Under the above assumptions and considerations every universal Faber-Laurent series $f \in U\left(\Omega,\left\{T_{n}\right\}\right)$ does not extend continuously on the point $z_{0} \in \partial \Omega$. In particular, $f$ cannot be holomorphically extended to any domain strictly containing $\Omega$.

For the proof it suffices to observe that if $f \in U\left(\Omega,\left\{T_{n}\right\}\right)$, then $f_{0}$ is a universal Faber series in $A_{0}^{\mathrm{c}}$ in the sense of $[4]$ and each $f_{j}, j \in\{1, \ldots, k\}$, is continuous on $\partial\left(A_{0}^{\mathrm{c}}\right)$. Thus, for $z_{0} \in \partial\left(A_{0}^{\mathrm{c}}\right)$ the existence of

$$
\lim _{\substack{\zeta \rightarrow z_{0} \\ z \in A_{0}^{c}}} f(z)
$$

is equivalent to that of

$$
\lim _{\substack{\zeta \rightarrow z_{0} \\ z \in A_{0}^{\mathrm{c}}}} f_{0}(z)
$$

But the last limit does not exist in $\mathbb{C}[\mathbf{4}, \mathbf{7}]$.

The proof for the other boundaries of $\Omega$ is similar.

Acknowledgements. We express our gratitude to the referee for helping us to improve considerably the presentation of this article.

\section{References}

1. G. Costakis and A. Melas, On the range of universal functions, Bull. Lond. Math. Soc. 32 (2000), 458-464.

2. W. Gehlen, W. Luh and J. MÜLler, On the existence of $O$-universal functions, Complex Variables 41 (2000), 81-90.

3. J.-P. Kahane, Baire's category theorem and trigonometric series, J. Analyse Math. 80 (2000), 143-182.

4. E. Katsoprinakis, V. Nestoridis and I. Papadoperakis, Universal Faber series, Analysis 21 (2001), 339-363.

5. A. I. Markushevich, Theory of functions of a complex variable, vol. III (Englewood Cliffs, NJ, Prentice-Hall, 1967).

6. A. Melas, On the growth of universal functions, J. Analyse Math. 82 (2000), 1-20.

7. A. Melas and V. Nestoridis, Universality of Taylor series as a generic property of holomorphic functions, Adv. Math. 157 (2001), 138-176. 
8. A. Melas, V. Nestoridis And I. Papadoperakis, Growth of coefficients of universal Taylor series and comparison of two classes of functions, J. Analyse Math. 73 (1997), 187-202.

9. D. Menchoff, Sur les séries trigonométriques universelles, Dokl. Akad. Nauk SSSR 49 (1945), 79-82.

10. C. Miranda, Sur un nouveau critére de normalité pour les familles de fonctions holomorphes, Bull. Soc. Math. France 63 (1935), 185-196.

11. V. Nestoridis, Universal Taylor series, Annls Inst. Fourier 46 (1996), 1293-1306.

12. V. Nestoridis, An extension of the notion of universal Taylor series, in Computational Methods and Function Theory, 1997, (CMFT 97), Nicosia, Cyprus, 1997, pp. 421-430 (World Scientific, 1999).

13. W. Rudin, Real and complex analysis (New York, McGraw-Hill, 1966).

14. V. Vlachou, Coincidence of two classes of universal Laurent series, Complex Variables 47 (2002), 1045-1053.

15. V. Vlachou, On some classes of universal functions, Analysis (Munich) 22(2) (2002), 149-161. 